\title{
Outcome of Resynchronization Therapy on Superficial and Endocardial Electrophysiological Findings
}

\author{
O. KITTNAR ${ }^{1}$, L. RIEDLBAUCHOVÁ ${ }^{2}$, T. ADLA ${ }^{3}$, V. SUCHÁNEK ${ }^{3}$, J. TOMIS $^{2}$, \\ M. LOŽEK ${ }^{4}$, A. VALERIÁNOVÁ ${ }^{1}$, M. HRACHOVINA ${ }^{1}$, M. POPKOVÁ ${ }^{1}$, J. VESELKA ${ }^{2}$, \\ J. JANOUŠEK ${ }^{4}$, L. LHOTSKÁ ${ }^{5}$
}

${ }^{1}$ Institute of Physiology, First Faculty of Medicine, Charles University in Prague, Czech Republic, ${ }^{2}$ Department of Cardiology, University Hospital in Motol and Second Faculty of Medicine, Charles University in Prague, Czech Republic, ${ }^{3}$ Department of Radiology, University Hospital in Motol, Czech Republic, ${ }^{4}$ Children's Heart Centre, Motol Children's Hospital, Prague, Czech Republic, ${ }^{5}$ The Czech Institute of Informatics, Robotics and Cybernetics, Czech Technical University, Prague, Czech Republic

Received March 14, 2018

Accepted October 2, 2018

\section{Summary}

Cardiac resynchronization therapy (CRT) has proven efficacious in the treatment of patients with heart failure and dyssynchronous activation. Currently, we select suitable CRT candidates based on the QRS complex duration (QRSd) and morphology with left bundle branch block being the optimal substrate for resynchronization. To improve CRT response rates, recommendations emphasize attention to electrical parameters both before implant and after it. Therefore, we decided to study activation times before and after CRT on the body surface potential maps (BSPM) and to compare thus obtained results with data from electroanatomical mapping using the CARTO system. Total of 21 CRT recipients with symptomatic heart failure (NYHA II-IV), sinus rhythm, and QRSd $\geq 150 \mathrm{~ms}$ and 7 healthy controls were studied. The maximum QRSd and the longest and shortest activation times (ATmax and ATmin) were set in the BSPM maps and their locations on the chest were compared with CARTO derived time interval and site of the latest (LATmax) and earliest (LATmin) ventricular activation. In CRT patients, all these parameters were measured during both spontaneous rhythm and biventricular pacing (BVP) and compared with the findings during the spontaneous sinus rhythm in the healthy controls. QRSd was $169.7 \pm 12.1$ ms during spontaneous rhythm in the CRT group and $104.3 \pm 10.2 \mathrm{~ms}$ after CRT $(p<0.01)$. In the control group the QRSd was significantly shorter: $95.1 \pm 5.6 \mathrm{~ms}(\mathrm{p}<0.01)$. There was a good correlation between LATmin(CARTO) and ATmin(BSPM). Both LATmin and ATmin were shorter in the control group (LATmin(CARTO) 24.8 $\pm 7.1 \mathrm{~ms}$ and ATmin(BSPM)
29.6 $\pm 11.3 \mathrm{~ms}, \mathrm{NS}$ ) than in CRT group (LATmin(CARTO) was $48.1 \pm 6.8 \mathrm{~ms}$ and ATmin(BSPM) $51.6 \pm 10.1 \mathrm{~ms}$, NS). BVP produced shortening compared to the spontaneous rhythm of CRT recipients (LATmin(CARTO) $31.6 \pm 5.3 \mathrm{~ms}$ and ATmin(BSPM) 35.2 $\pm 12.6 \mathrm{~ms} ; \mathrm{p}<0.01$ spontaneous rhythm versus BVP). ATmax exhibited greater differences between both methods with higher values in BSPM: in the control group LATmax(CARTO) was $72.0 \pm 4.1 \mathrm{~ms}$ and ATmax (BSPM) $92.5 \pm 9.4 \mathrm{~ms}(p<0.01$ ), in the CRT candidates LATmax(CARTO) reached only $106.1 \pm 6.8 \mathrm{~ms}$ whereas ATmax(BSPM) $146.0 \pm 12.1 \mathrm{~ms}(\mathrm{p}<0.05)$, and BVP paced rhythm in CRT group produced improvement with LATmax(CARTO) $92.2 \pm 7.1 \mathrm{~ms}$ and ATmax(BSPM) $130.9 \pm 11.0 \mathrm{~ms}$ $(p<0.01$ before and during BVP). With regard to the propagation of ATmin and ATmax on the body surface, earliest activation projected most often frontally in all 3 groups, whereas projection of ATmax on the body surface was more variable. Our results suggest that compared to invasive electroanatomical mapping BSPM reflects well time of the earliest activation, however provides longer time-intervals for sites of late activation. Projection of both early and late activated regions of the heart on the body surface is more variable than expected, very likely due to changed LV geometry and interposed tissues between the heart and superficial ECG electrode.

\section{Key words}

Cardiac resynchronization therapy • Heart failure • Dyssynchrony - Electrocardiography • Body surface mapping • Biventricular pacing 


\section{Corresponding author}

O. Kittnar, Institute of Physiology, Charles University in Prague, First Faculty of Medicine, Albertov 5, 12800 Prague 2, Czech Republic. E-mail: otomar.kittnar@lf1.cuni.cz

\section{Introduction}

Cardiac resynchronization therapy (CRT) has proven efficacious in the treatment of patients with heart failure and dyssynchronous activation. Currently, we select suitable CRT candidates based on the presence of electrical asynchrony, i.e. based on the QRS complex duration and morphology with left bundle branch block (LBBB) being the most common substrate for CRT (Ponikowski et al. 2016, De Pooter et al. 2018). Altered velocity and uniformity of depolarization waveform propagation in the dyssynchronous heart causes delayed electrical (and presumably also mechanical) activation of some left ventricular areas which may participate on the further progression of heart failure. However, the QRS width reflects rather a total duration of ventricular activation and doesn't provide information about the space and temporal characteristics of the ventricular activation pattern (Fauchier et al. 2002). Moreover, it has been shown that ventricular activation patterns may be individually variable in patients with electrocardiographic morphology of LBBB. This fact has revived interest in the ventricular activation sequence related to the LBBB as this may be associated with heterogeneity in the mechanical dyssynchrony among patients with LBBB which could be one of the reasons why significant number of patients fails to respond to CRT (Auricchio et al. 2004).

Although the goal of CRT is to ensure more coordinated contraction pattern, our current possibilities to detect and quantify mechanical dyssynchrony in CRT candidates are still only limited. Therefore we presume that the presence and degree of mechanical dyssynchrony correspond with the severity of electrical asynchrony. However, standard 12-lead ECG activation assessment of the left ventricle (LV) with LBBB morphology provides only a gross estimate of the activation sequence in the dilated heart and thus it cannot give reasonable information about activation times at different ventricular sites. To improve CRT response rates, recommendations emphasize attention to electrical parameters both before implant and either directly at implant during left ventricular lead positioning or immediately after it (Varma et al. 2018). Therefore, we decided to study activation times before and after CRT on the body surface potential maps (BSPM) that may serve as a noninvasive tool for assessment of ventricular activation sequence. To validate observations from BSPM with regard to the location and time of the earliest and latest ventricular activation, we compared BSPM results with data that were obtained during invasive electroanatomical mapping using the CARTO system.

\section{Methods and patients}

Electrocardiographic recordings were obtained from 21 patients ( 12 men, 9 women, age $61.5 \pm 6.1$ years) who underwent implantation of CRT pacemaker/ defibrillator in accordance with current guidelines, i.e. symptomatic HF in New York Heart Association (NYHA) functional class II to ambulatory IV despite adequate medical treatment, left ventricular ejection fraction $\leq 35 \%$, sinus rhythm, intraventricular conduction disturbance with QRS duration $\geq 120 \mathrm{~ms}$ in LBBB type or $\geq 150 \mathrm{~ms}$ in nonLBBB type. Demographic description of the study population and response to CRT are summarized in Tables 1 and 2.

To compare findings in CRT recipients with those observed in healthy subject, the same electrocardiographic recordings were obtained during spontaneous rhythm from the control group containing 7 healthy patients ( 2 men, 5 women, age $47.5 \pm 11.3$ years) who underwent invasive CARTO mapping before ablation of their benign ventricular ectopy originating in the right ventricular outflow tract. A healthy person was defined for the purpose of this project according to the following findings and data: no structural cardiac disease (confirmed by magnetic resonance imaging and echocardiography (ECHO)), and sinus rhythm with normal morphology of heart beats on ECG.

Electrocardiographic and BSPM recordings were obtained simultaneously using the BioSemi device as previously described (Kittnar et al. 2017). The examination was performed under standard conditions. Two minutes were recorded during spontaneous rhythm in both CRT recipients and healthy controls and during biventricular pacing (BVP) in the CRP group. Following parameters were evaluated: the longest QRS complex duration (QRSd) detected in any BSPM lead, the shortest and the longest activation time (ATmin and ATmax) and locations of the ATmin and ATmax on the body surface. The activation time (AT) was defined as the duration between the QRS onset and the steepest negative slope of 
the unipolar QRS complex (minimum dV/dt) (Fig. 1) (Akahoshi et al. 1997, Bank et al. 2017). ATmax was defined as the longest and ATmin as the shortest AT interval detected in any of the 123 unipolar chest leads used for BSPM. The parameters were labeled manually using a graphic system developed for this purpose in Matlab 8.0 programming environment.
A section of the recording with constant R-R intervals and morphology was selected and an average beat was calculated for each lead. Parameters were then labeled from the average beat. Before labelling, signals were preprocessed using standard filtration $(0.67 \mathrm{~Hz}$ highpass and $45 \mathrm{~Hz}$ lowpass FIR filters, $50 \mathrm{~Hz}$ notch filter and DC offset removal).

Table 1. Baseline characteristic of the study population (smaller size of "CARTO" group is caused either by the disagreement of the patient to undergo invasive procedure or by the suboptimal results of the CARTO mapping that couldn't be used for the purpose of this study).

\begin{tabular}{|c|c|c|}
\hline BSPM & CRP group & Control group \\
\hline Number of patients & 21 & 7 \\
\hline Men / Women & $12 / 9$ & $2 / 5$ \\
\hline Age (years) & $61.5 \pm 6.1$ & $47.5 \pm 11.3$ \\
\hline$B M I$ & $28.0 \pm 4.6$ & $26.3 \pm 3.1$ \\
\hline NYHA class & $2.5 \pm 0.5$ & 1 \\
\hline ECHO: LVEF (\%) & $25 \pm 8$ & $60 \pm 6$ \\
\hline $\operatorname{LVESV}(m l)$ & $141 \pm 56$ & $38 \pm 9$ \\
\hline$L V E D V(m l)$ & $184 \pm 62$ & $92 \pm 11$ \\
\hline $\begin{array}{l}\text { Type of conduction delay: } \\
L B B B / \text { non } L B B B(L B B B \text {-like) }\end{array}$ & $11 / 10$ & Normal QRS \\
\hline CARTO & CRP group & Control group \\
\hline Number of patients & 11 & 3 \\
\hline Men / Women & $6 / 5$ & $1 / 2$ \\
\hline Age (years) & $62.8 \pm 8.3$ & $53.7 \pm 4.3$ \\
\hline NYHA class & $2.5 \pm 0.5$ & 1 \\
\hline$B M I$ & $27.7 \pm 4.8$ & $26.1 \pm 4.2$ \\
\hline ECHO: LVEF (\%) & $23 \pm 8$ & $60 \pm 2$ \\
\hline $\operatorname{LVESV}(m l)$ & $148 \pm 56$ & $38 \pm 9$ \\
\hline$L V E D V(m l)$ & $190 \pm 60$ & $101.1 \pm 9.6$ \\
\hline $\begin{array}{l}\text { Type of conduction delay: } \\
L B B B / \text { non } L B B B(L B B B \text {-like) }\end{array}$ & $9 / 2$ & Normal QRS \\
\hline
\end{tabular}

BMI - body mass index, ECHO-LVEF - ejection fraction of the left ventricle assessed by 2D echocardiography, ECHO-LVEDV and LVESV, resp. left-ventricular enddiastolic and endsystolic, resp. volume assessed by $2 \mathrm{D}$ echocardiography.

Table 2. Clinical outcome after 6 months of CRT.

\begin{tabular}{cccc}
\hline & Baseline & 6 months follow-up & p value \\
\hline NYHA class & $2.3 \pm 0.6$ & $1.6 \pm 0.6$ & $<0.001$ \\
ECHO: LVEF $(\%)$ & $24 \pm 8$ & $30 \pm 8$ & $<0.05$ \\
LVESV $(\mathrm{ml})$ & $141 \pm 56$ & $136 \pm 92$ & 0.82 \\
LVEDV $(\mathrm{ml})$ & $184 \pm 62$ & $186 \pm 104$ & 0.93 \\
NT pro BNP $(\mathrm{ng} / \mathrm{l})$ & $2,426 \pm 2,631$ & $1,589 \pm 2,772$ & 0.23 \\
\hline
\end{tabular}

Abbreviations as in Table 1. 

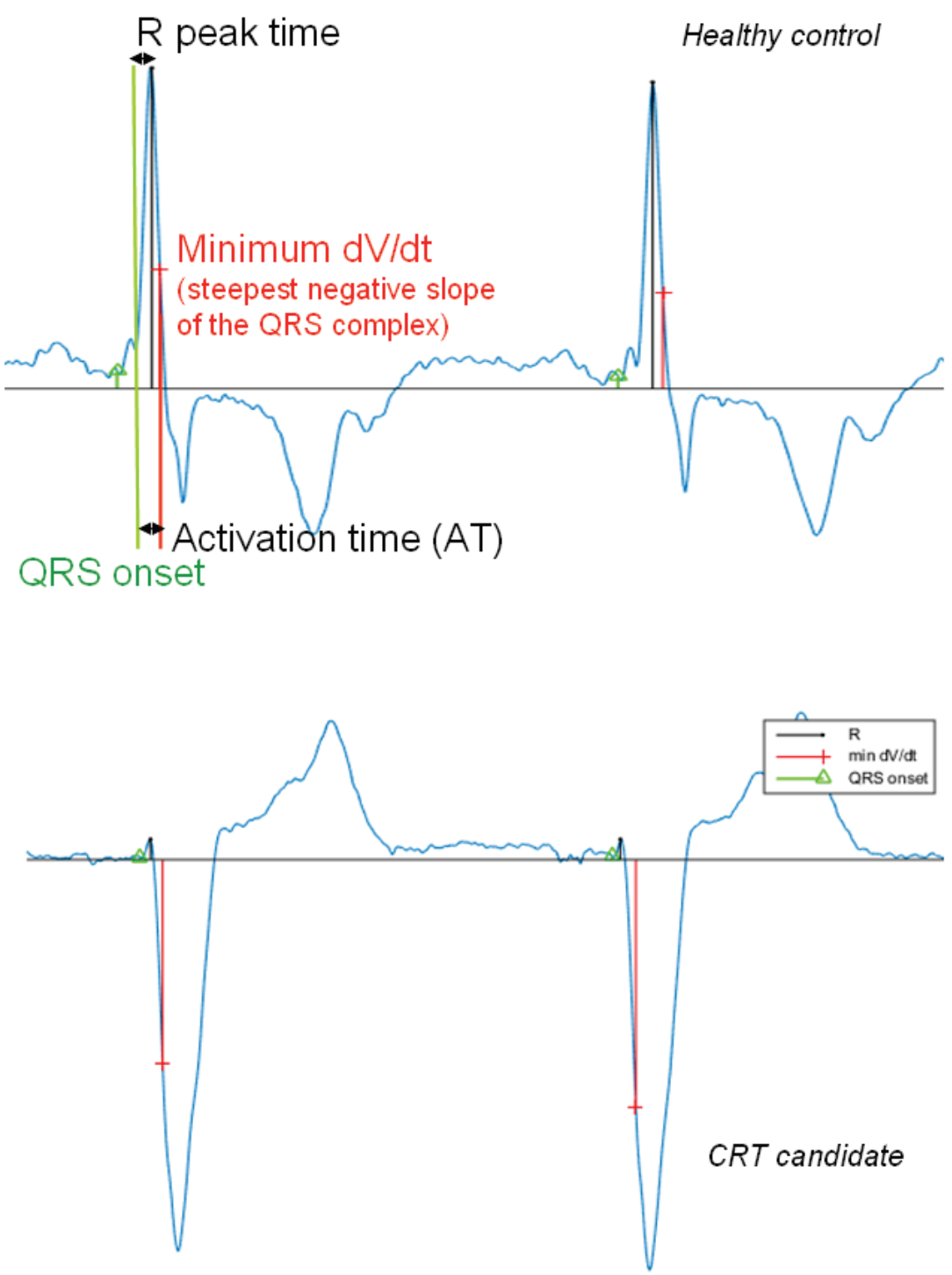

Fig. 1. Activation time (AT) was set as the time interval between onset of the QRS complex (green line) and the minimum $\mathrm{dV} / \mathrm{dt}$ on the QRS wave (red line), black line corresponds to the maximum amplitude of the QRS complex.
Data derived from the BSPM maps were then compared with those acquired during invasive activation mapping using CARTO3 system, because it provides detail information with regard to the individual activation sequence of both ventricles that cannot be derived in such an extent solely from the standard 12-lead ECG. CARTO recordings were obtained during spontaneous rhythm in both study groups and during BVP in CRT recipients as well. Both BSPM and CARTO mapping were performed 3-5 weeks after CRT implantation. Before CARTO mapping, the CRT system was inactive. After acquisition of ventricular activation maps during spontaneous rhythm, CRT system was fully activated and remap of both ventricles was made during final BVP programming. In the control group, CARTO mapping of both ventricles during sinus rhythm was part of the diagnostic procedure, before identification of the focus of ventricular ectopy and its ablation were performed. Mean of 290 points were recorded in left versus 220 points in the right ventricle.
However, not all CRT patients gave their informed consent and underwent invasive CARTO mapping and not all CARTO recording were suitable for further analysis. Full CARTO data were thus available in 11 patients from the CRT group ( 6 men, 5 women, age $62.8 \pm 8.3$ years) and in 3 patients from the control group ( 1 man, 2 women, age $53.7 \pm 4.3$ years) (Table 1 ). The following parameters were derived from the activation CARTO maps: local activation time (LAT) of each point was set as the time from the QRS onset on the superficial ECG tracing to the first high-frequency component of the bipolar signal and/or maximum negative slope of the appropriate unipolar signal. Sites with low voltage $(<0.2 \mathrm{mV})$ and absence of pacing capture were marked as the dense scar. The time LATmin referred to the shortest LAT while LATmax to the longest LAT (Fig. 2).

Statistical comparison was performed using the nonparametric Mann-Whitney test. 

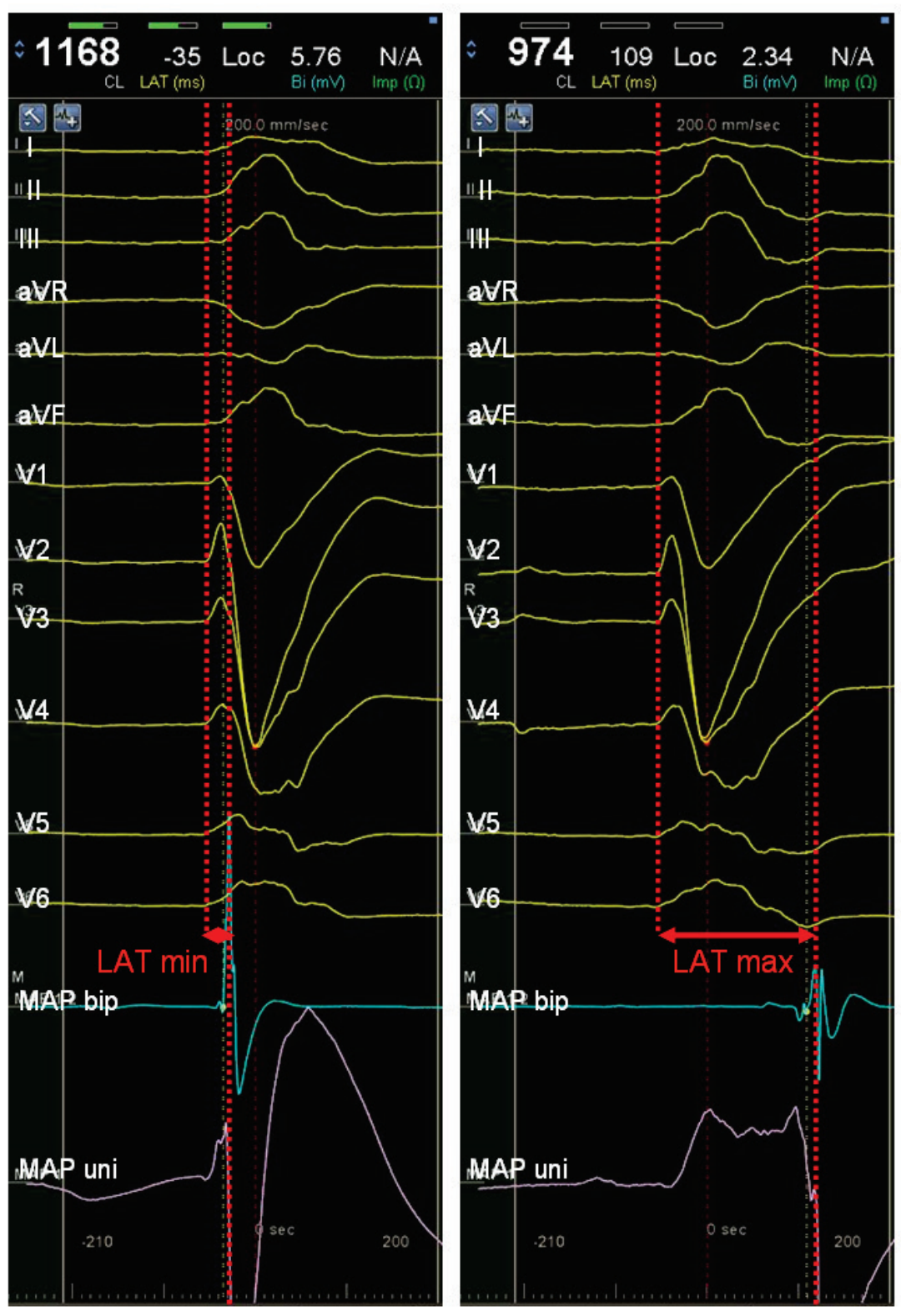

Fig. 2. Local activation time (LAT) was set as the time interval between onset of the QRS complex and local electrogram that was registered at each individual point of the CARTO map. LATmin reflected point with the shortest LAT, on the contrary LATmax was the point with the longest LAT among the appropriate ventricle (left versus right).

\section{Results}

Clinical outcome after 6 months of BVP is summarized in Table 2. Since not all CRT patients already reached their 6 months follow-up visit at the time of results publication, the degree of CRT-induced improvement didn't reach statistical significance in all parameters. However, the trend towards regression of the left-ventricular dilatation and decrease in natriuretic peptides were detectable in spite of this fact.

All findings with regard to BSPM and CARTO results are summarized in Figure 3. Duration of the QRS complex (QRSd) was $169.7 \pm 12.1 \mathrm{~ms}$ in the group of patients with spontaneous rhythm before CRT and $104.3 \pm 10.2 \mathrm{~ms}$ after CRT $(\mathrm{p}<0.01)$. In the control group the QRSd was significantly shorter as compared to both measurements in CRT group: 95.1 $\pm 5.6 \mathrm{~ms}(\mathrm{p}<0.01)$.

There was a good correlation between variables describing the earliest activation of the ventricles during the spontaneous rhythm, it is between LATmin(CARTO) and ATmin(BSPM). As expected, both LATmin and ATmin were much shorter in the control group: LATmin(CARTO) was $24.8 \pm 7.1 \mathrm{~ms}$ and ATmin(BSPM) $29.6 \pm 11.3 \mathrm{~ms}$ in the control group (NS), while in the CRT group LATmin(CARTO) was $48.1 \pm 6.8 \mathrm{~ms}$ and ATmin(BSPM) $51.6 \pm 10.1 \mathrm{~ms}$ (NS). BVP produced shortening compared to the spontaneous rhythm, but not normalization of the time of the earliest activation: LATmin(CARTO) during BVP was $31.6 \pm 5.3 \mathrm{~ms}$ and ATmin(BSPM) during BVP $35.2 \pm 12.6 \mathrm{~ms}$ (NS). Significant decrease-producing effect of BVP on ATmin was found by both measuring methods ( $p<0.01)$. 

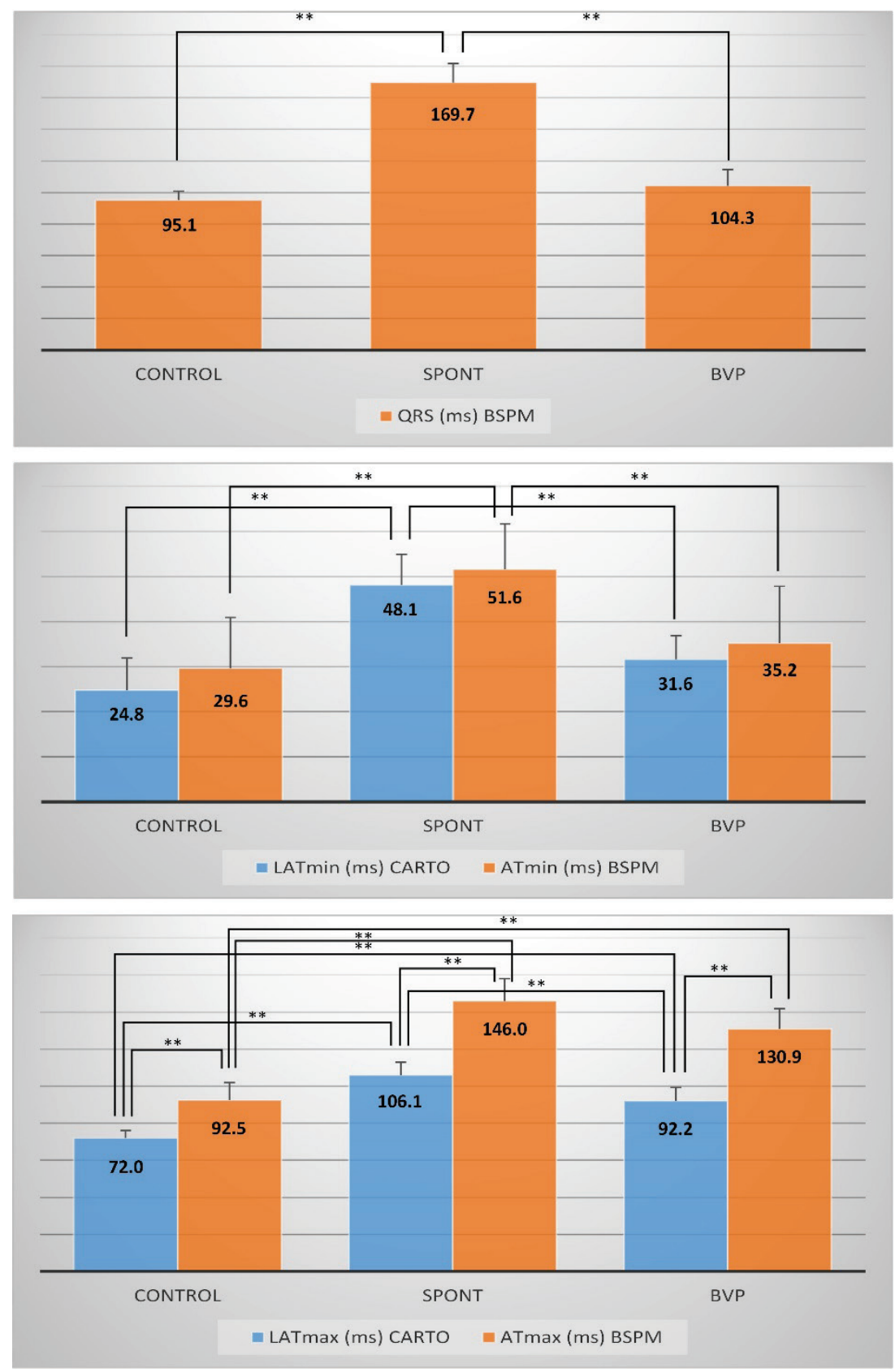

Fig. 3. Graphs of the results. Upper graph: width of the QRS complex (QRS) in milliseconds (ms), middle graph: the shortest activation time (ATmin, ms), lower graph: the longest activation time (ATmax, ms), CONTROL - spontaneous rhythm in control group (healthy subjects), SPONT - spontaneous rhythm in CRT recipients; BVP - biventricular pacing rhythm in CRT recipients.
On the other hand, variables describing the time of the latest activation exhibited greater difference between both methods with higher values in BSPM method: 1) spontaneous rhythm in the control group: LATmax (CARTO) 72.0 $\pm 4.1 \mathrm{~ms}$ and ATmax (BSPM) $92.5 \pm 9.4 \mathrm{~ms}(\mathrm{p}<0.01), 2)$ spontaneous rhythm in CRT patients: LATmax (CARTO) 106.1 $\pm 6.8 \mathrm{~ms}$ and ATmax (BSPM) 146.0 $\pm 12.1 \mathrm{~ms}(\mathrm{p}<0.05)$, and 3) BVP paced rhythm in CRT patients: LATmax (CARTO) $92.2 \pm 7.1 \mathrm{~ms}$ and ATmax (BSPM) 130.9 $\pm 11.0 \mathrm{~ms}(\mathrm{p}<0.01)$. Similarly as with the earliest activation, BVP produced improvement of time to the latest activation (as assessed by both methods). Although shortening of this time interval was significant during BVP, neither ATmax, nor LATmax reached values of the control group.

With regard to the location of the early and late activated regions during spontaneous rhythm, significant differences were found between groups (Fig. 4):

In the control group, site of the early activation on CARTO maps corresponded with the terminal branching of the conduction system, thus LATmin for the right ventricle (RV LATmin) and left ventricle (LV LATmin) were found on the interventricular septum and on the LV lateral free wall. Corresponding ATmin on BSPM projected to the middle frontal region of the chest (Fig. 4A). In CRT candidates, the earliest activation was 
shifted towards the RV with its free wall being activated first, usually at the same time as the middle part of the interventricular septum based on the CARTO maps. ATmin projection on the chest was generally similar in these patients as in the control group (Fig. 4B). Compared to the spontaneous rhythm, site of the earliest activation during BVP differed inter-individually and corresponded to location of the pacing site with basal or middle part of the LV free wall and $\mathrm{mid} /$ apical interventricular septum being the most common sites of the earliest activation of LV and RV, resp. on CARTO. Although projection of the earlies activation (ATmin) on the body surface during BVP was also more variable, most often it was also located frontally (Fig. 4C).

Localization of the late activation was similar in both healthy controls and CRT candidates and was present on the basal LV lateral wall (Fig. 4A, B). However, projection of the site of late activation (ATmax(BSPM)) differed between these groups with its location on the back in the control, but more variably in the CRT candidates with some cases of frontal projection. Similarly as with ATmin and LATmin, also distribution of late activation during BVP was more inter-individually variable as it was influenced by the programming of atrioventricular delay, degree of LV preexcitation by the LV pacing lead and the degree of fusion with the spontaneous AV conduction. In most cases was the site of late activation located in the anteroseptal or inferoseptal regions of LV basally based on the CARTO maps with projection of these regions most often posteriorly close to the left shoulder on the body surface (Fig. 4C).

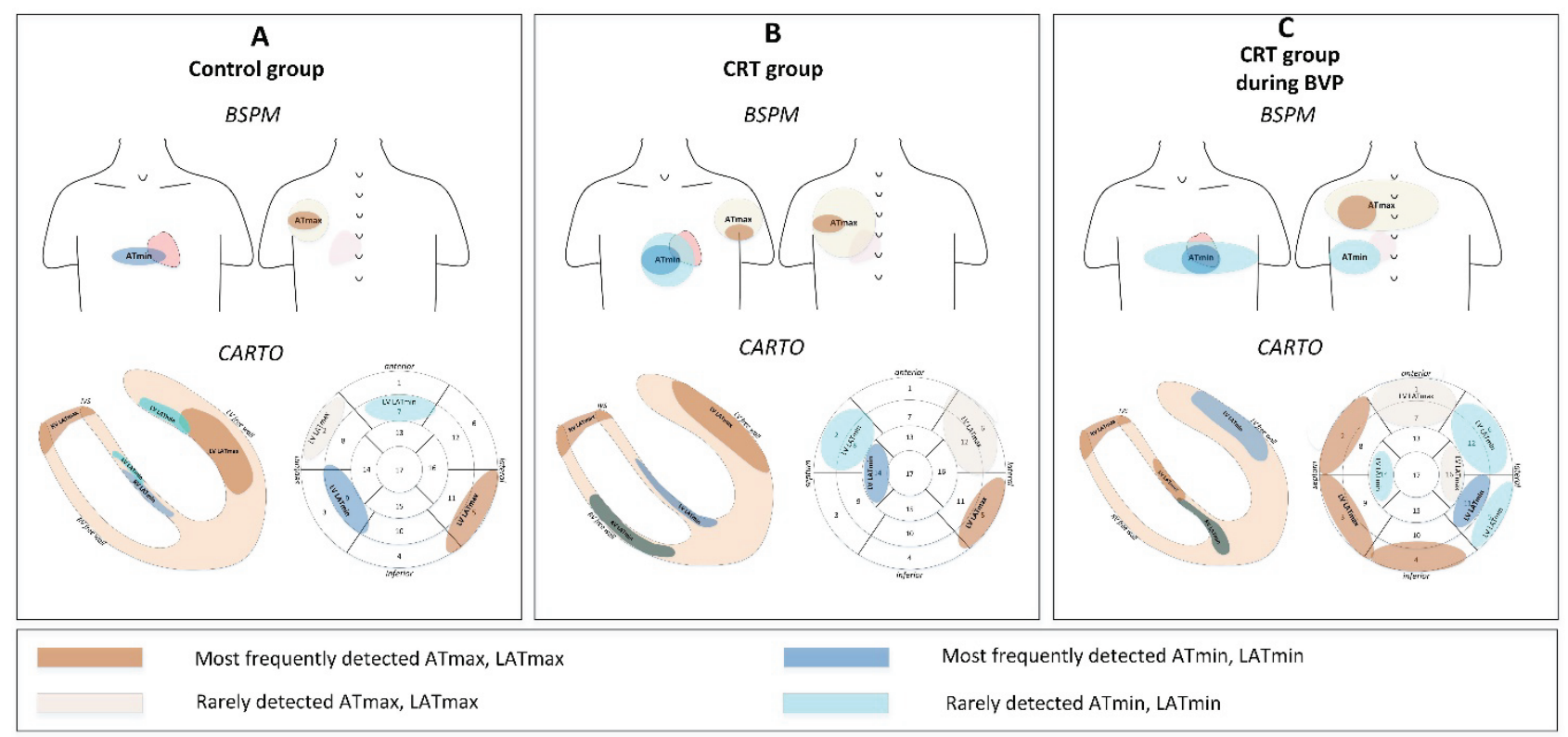

Fig. 4. Distribution of the shortest and longest activation times in control group, in CRT recipients during spontaneous rhythm and during BVP. Upper portion: BSPM projections of ATmin and ATmax to the chest surface. Lower left portion: CARTO projections of LATmin and LATmax of both ventricles in horizontal view (LV and RV resp.), IVS=interventricular septum. Lower right portion: CARTO projections of LATmin and LATmax of the left ventricle to the left ventricular segmentation according to American Heart Association (Cerqueira et al. 2002): 1-basal anterior, 2-basal anteroseptal, 3-basal inferoseptal, 4-basal inferior, 5-basal inferolateral, 6-basal anterolateral, 7-mid anterior, 8-mid anteroseptal, 9-mid inferoseptal, 10-mid inferior, 11-mid inferolateral, 12-mid anterolateral, 13-apical anterior, 14-apical septal, 15-apical inferior, 16-apical lateral, 17-apex.

\section{Discussion}

The present study confirmed observation of others that patients with LBBB morphology have specific activation sequence that differs significantly from the physiologic. However, conventional ECG activation mapping in the dilated human left ventricle with LBBB morphology achieves only a gross estimate of the electrical activation sequence giving the limited number of recording sites that may be collected. That is why the BSPM can bring more detailed information as a higher number of recording sites can significantly increase a possibility to identify more preciously both the value and site of the shortest and longest activation time measured from the body surface (Kittnar and Stovicek 1993, Kittnar and Mlcek 2010). Big advantage of activation time measurement is that this parameter is not sex-related (Waldeyer et al. 2009). On the other hand, its 
crucial disadvantage is that it depends on the age: it was proved that activation times measured from the surface electrocardiography are longer in older hearts (Waldeyer et al. 2009, Laszlo et al. 2017).

Our data show that CRT may induce significant shortening of the QRS duration as well as of the time to the earliest and latest ventricular activation. This effect of CRT was found identically both from the endocardium (CARTO mapping) and from the body surface (BSPM). With this regard, this result of our study is not very surprising, however. Contemporarily, CRT is the only clinical treatment that can both acutely and chronically modify electrical activation pattern of the ventricles and thus improve systolic function (Kirk and Kass 2013). However, one must be cautious with extrapolation that observed electrical resynchronization leads to similar mechanical resynchronization. It applies especially to the shortening of the QRS complex. Although the duration of spontaneous QRS complex is used as a measure of particularly left ventricular dyssynchrony to select patients for CRT (Dickstein et al. 2010), it was shown that poor correlation between the QRS width and the mechanical dyssynchrony exists (Auger et al. 2012, Bleeker et al. 2004). On the other hand, importance of QRSd was suggested by the finding that LV reverse remodeling with CRT occurs at smaller QRSd (Linde et al. 2018).

Even more complicated is the interpretation of activation times, particularly the ATmax. We have observed longer maximum activation time in BSPM (ATmax) than in CARTO maps (LATmax). However, minimum activation times corresponded well between both methods. Possible explanation of this finding might be that electroanatomical CARTO mapping records "only" endocardial activation of the ventricles, whereas BSPM reflects the course of depolarization throughout the entire ventricular wall, including lately activated subepicardial regions that are omitted by CARTO. On the other hand, early activation occurs constantly endocardially, thus the correlation between ATmin and LATmin is very close in this case. We assume that higher values of ATmax (BSPM) compared to LATmax (CARTO) should be taken into account, particularly if activation times are suggested to be a possible marker of a CRT success rate. Namely Sweeney et al. (2010) have shown that patients with shorter LV activation time delays $(\leq 80 \mathrm{~ms}$ ) had a $51 \%$ response rate to CRT compared with $73 \%$ response in patients with larger LV activation time delays ( $\geq 125 \mathrm{~ms}$ ). These differences in LV activation time delays may result in different LV mechanical activation patterns that determine a different response to CRT (Auger et al. 2012). We assume that ATmax might be better marker both of the dyssynchrony extent and possibly of the CRT effect than the QRS duration although this hypothesis has to be tested in a larger study yet.

Partially surprising were the results of projection of the site of early and late activation on the body surface. While early activation was found in the middle frontal level of the chest in all three groups (including BVP where LV free wall was artificially preexcitated by the pacing lead). projections of ATmax differed significantly between health controls, CRT candidates and during BVP. Possible explanation of these findings might be that ATmax reflects events that happen in the entire heart, including the LV epicardium as well as activity of the right ventricle at the same time, whereas CARTO reflects only endocardial activation of the appropriate chamber. In addition, in patients with advanced heart failure and intraventricular conduction disturbance, cardiac remodeling often results in balloon-like change of the left-ventricular shape, but it may also modify the position of the heart in the thorax with cardiac apex pointing more into the axilla. Moreover, projection of the electrical processes from endocardium on the body surface is complicated by many tissues lying between the heart and the body surface and different electrical conductivity of various tissues. Thus locations of the early and late activated regions of the LV on the body surface after CRT could be a little bit surprising in comparison with usual CARTO findings.

\section{Study limitations}

The crucial limitation is relatively small number of patients included in the study. However, CARTO mapping represents time-demanding procedure that carries also certain risks attributed to its invasive nature. Therefore it is difficult to obtain data on high volume of patients to compare findings on invasive CARTO mapping with body-surface projection of the electrical events and our data should be viewed as unique with this regard.

\section{Conclusions}

Our results suggest that compared to invasive electroanatomical mapping BSPM reflects well time of 
the earliest activation, however provides longer timeintervals for sites of late activation. The greater values of ATmax (BSPM) in contrast to LATmax (CARTO) are very likely attributed to the fact that BSPM mirrors events of the entire wall and heart as compared to CARTO, whereas both these methods detect similarly well the moment of the earliest activation.

It seems that projection of both early and late activated regions of the heart on the body surface is sometimes modified by the changed LV geometry and interposed tissues between the heart and superficial ECG electrode.

\section{Conflict of Interest}

There is no conflict of interest.

\section{Acknowledgements}

Supported by Ministry of Health of the Czech Republic, grant Nr. 15-31398A and SVV 260379. All rights reserved.

\section{References}

AKAHOSHI M, HIRAI M, INDEN Y, SANO H, SHIMIZU A, KONDO T, MAKINO M, HORIBA M, YOSHIDA Y, TSUBOI N, HIRAYAMA H, ITO T, HAYASHI H, SAITO H: Body-surface distribution of changes in activation-recovery intervals before and after catheter ablation in patients with Wolff-Parkinson-White syndrome: clinical evidence for ventricular 'electrical remodeling' with prolongation of action-potential duration over a preexcited area. Circulation 96: 1566-1574, 1997.

AUGER D, BLEEKER GB, BERTINI M, EWE SH, VAN BOMMEL RJ, WITKOWSKI TG, NG AC, VAN ERVEN L, SCHALIJ MJ, BAX JJ, DELGADO V: Effect of cardiac resynchronization therapy in patients without left ventricular dyssynchrony. Eur Heart J 33: 913-920, 2012.

AURICCHIO A, FANTONI C, REGOLI F, CARBUCICCHIO C, GOETTE A, GELLER C, KLOSS M, KLEIN H: Characterization of left ventricular activation in patients with heart failure and left bundle-branch block. Circulation 109: 1133-1139, 2004.

BANK AJ, GAGE RM, CURTIN AE, BURNS KV, GILLBERG JM, GHOSH S: Body surface activation mapping of electrical dyssynchrony in cardiac resynchronization therapy patients: potential for optimization. J Electrocardiol 51: 534-541, 2018.

BLEEKER GB, SCHALIJ MJ, MOLHOEK SG, VERWEY HF, HOLMAN ER, BOERSMA E, STEENDIJK P, VAN DER WALL EE, BAX JJ: Relationship between QRS duration and left ventricular dyssynchrony in patients with end-stage heart failure. J Cardiovasc Electrophysiol 15: 544-549, 2004.

CERQUEIRA MD, WEISSMAN NJ, DILSIZIAN V, JACOBS AK, KAUL S, LASKEY WK, PENNELL DJ, RUMBERGER JA, RYAN T, VERANI MS, AMERICAN HEART ASSOCIATION WRITING GROUP ON MYOCARDIAL SEGMENTATION AND REGISTRATION FOR CARDIAC IMAGING: Standardized myocardial segmentation and nomenclature for tomographic imaging of the heart. A statement for healthcare professionals from the Cardiac Imaging Committee of the Council on Clinical Cardiology of the American Heart Association. Circulation 105: 539-542, 2002.

De POOTER J, El HADDAD M, KAMOEN V, KALLUPURACKAL TT, STROOBANDT R, DE BUYZERE M, TIMMERMANS F: Relation between electrical and mechanical dyssynchrony in patients with left bundle branch block: An electro- and vectorcardiographic study. Ann Noninvasive Electrocardiol 23: e12525, 2018.

DICKSTEIN K, VARDAS PE, AURICCHIO A, DAUBERT JC, LINDE C, MCMURRAY J, PONIKOWSKI P, PRIORI SG, SUTTON R, VAN VELDHUISEN DJ, VAHANIAN A, BAX J, CECONI C, DEAN V, FILIPPATOS G, FUNCK-BRENTANO C, HOBBS R, KEARNEY P, MCDONAGH T, POPESCU BA, REINER Z, SECHTEM U, SIRNES PA, TENDERA M, VARDAS P, WIDIMSKY P, ANKER SD, BLANC JJ, GASPARINI M, HOES AW, ISRAEL CW, KALARUS Z, MERKELY B, SWEDBERG K, CAMM AJ: 2010 Focused Update of ESC Guidelines on device therapy in heart failure: an update of the 2008 ESC Guidelines for the diagnosis and treatment of acute and chronic heart failure and the 2007 ESC guidelines for cardiac and resynchronization therapy. Developed with the special contribution of the Heart Failure Association and the European Heart Rhythm Association. Eur Heart J 31: 2677-2687, 2010. 
FAUCHIER L, MARIE O, CASSET-SENON D, BABUTY D, COSNAY P, FAUCHIER JP: Interventricular and intraventricular dyssynchrony in idiopathic dilated cardiomyopathy: a prognostic study with Fourier phase analysis of radionuclide scintigraphy. J Am Coll Cardiol 40: 2022-2030, 2002.

KIRK JA, KASS DA: Electromechanical dyssynchrony and resynchronization of the failing heart. Circ Res 113: 765-776, 2013.

KITTNAR O, MLČEK M: Analysis of the electrical heart field. Physiol Res 59 (Suppl 1): S19-S24. 2010.

KITTNAR O, RIEDLBAUCHOVÁ L, TOMIS J, LOŽEK M, VALERIÁNOVÁ A, HRACHOVINA M, MLČEK M, HUPTYCH M, JANOUŠEK J, LHOTSKÁ L: Electrocardiographic outcome of resynchronization therapy. Physiol Res 66 (Suppl 4): S523-S528, 2017.

KITTNAR O, ŠŤOVÍČEK P: Contemporary body surface potential mapping in electrocardiology and its perspectives. Physiol Res 42: 141-143, 1993.

LASZLO R, KONZ H, KUNZ K, DALLMEIER D, KLENK J, DENKINGER M, KOENIG W, ROTHENBACHER D, STEINACKER JM FOR THE ACTIFE STUDY GROUP: Evaluation of left and right ventricular systolic and diastolic electromechanical synchrony in older people: a population-based observational study. Physiol Res 66 : 933-948, 2017.

LINDE C, CLELAND JGF, GOLD MR, CLAUDE DAUBERT J, TANG ASL, YOUNG JB, SHERFESEE L, ABRAHAM WT: The interaction of sex, height, and QRS duration on the effects of cardiac resynchronization therapy on morbidity and mortality: an individual-patient data meta-analysis. Eur J Heart Fail 20: 780-791, 2018.

PONIKOWSKI P, VOORS AA, ANKER SD, BUENO H, CLELAND JG, COATS AJ, FALK V, GONZÁLEZJUANATEY JR, HARJOLA VP, JANKOWSKA EA, JESSUP M, LINDE C, NIHOYANNOPOULOS P, PARISSIS JT, PIESKE B, RILEY JP, ROSANO GM, RUILOPE LM, RUSCHITZKA F, RUTTEN FH, VAN DER MEER P: 2016 ESC Guidelines for the diagnosis and treatment of acute and chronic heart failure: The Task Force for the diagnosis and treatment of acute and chronic heart failure of the European Society of Cardiology (ESC). Developed with the special contribution of the Heart Failure Association (HFA) of the ESC. Eur J Heart Fail 18: 891-975, 2016.

SWEENEY MO, VAN BOMMEL RJ, SCHALIJ MJ, BORLEFFS CJ, HELLKAMP AS, BAX JJ: Analysis of ventricular activation using surface electrocardiography to predict left ventricular reverse volumetric remodeling during cardiac resynchronization therapy. Circulation 121: 626-634, 2010.

VARMA N, O'DONNELL D, BASSIOUNY M, RITTER P, PAPPONE C, MANGUAL J, CANTILLON D, BADIE N, THIBAULT B, WISNOSKEY B: Programming cardiac resynchronization therapy for electrical synchrony: reaching beyond left bundle branch block and left ventricular activation delay. J Am Heart Assoc 7: pii: e007489, 2018.

WALDEYER C, FABRITZ L, FORTMUELLER L, GERSS J, DAMKE D, BLANA A, LAAKMANN S, KREIENKAMP N, VOLKERY D, BREITHARDT G, KIRCHHOF P: Regional, age-dependent, and genotype-dependent differences in ventricular action potential duration and activation time in 410 Langendorff-perfused mouse hearts. Basic Res Cardiol 104: 523-533, 2009. 